\title{
The value of ${ }^{18} \mathrm{~F}$-FDG PET/CT in the prediction of clinical outcomes of patients with acute leukemia treated with allogeneic hematopoietic stem cell transplantation
}

\author{
ZIXUAN ZHAO $^{1 *}$, YEYE ZHOU ${ }^{1 *}$, JING WANG $^{2}$, TONGTONG ZHANG $^{2}$, \\ JIHUI LI ${ }^{1}$, BIN ZHANG ${ }^{1}$, QINGRU LI ${ }^{1}$ and SHENGMING DENG ${ }^{1}$ \\ Departments of ${ }^{1}$ Nuclear Medicine and ${ }^{2}$ Hematology, The First Affiliated Hospital of Soochow University, \\ Suzhou, Jiangsu 215006, P.R. China
}

Received January 17, 2020; Accepted June 12, 2020

DOI: $10.3892 / \mathrm{ol} .2020 .12036$

\begin{abstract}
The present study aimed to determine whether ${ }^{18} \mathrm{~F}-\mathrm{FDG}$ PET/CT performed before and/or after allogeneic hematopoietic stem cell transplantation (allo-HSCT) can predict clinical outcomes in acute leukemia (AL). A total of 79 examinations comprising 72 patients with AL who underwent ${ }^{18} \mathrm{~F}$-FDG PET/CT before and/or after allo-HSCT were retrospectively enrolled between January 2011 and January 2019. Outcomes were assessed using overall survival (OS) and disease-free survival (DFS). A total of 63 examinations were PET-positive, while 16 examinations were PET-negative. Increased $\mathrm{BM}$ and splenic ${ }^{18} \mathrm{~F}$-FDG uptake were observed in $24(19 / 79)$ and 14\% (11/79) of examinations, respectively. ${ }^{18} \mathrm{~F}-\mathrm{FDG}$-avid lymph nodes were observed in 38\% (30/79) of examinations. $\mathrm{E}_{\mathrm{N}} \mathrm{E}_{\mathrm{M}} \mathrm{E}_{\mathrm{S}}$ involvement was detected in $44 \%$ $(35 / 79)$ of examinations. The presence of $E_{N} E_{M} E_{S}$ involvement [OS hazard ratio (HR), 6.399; 95\% confidence interval (CI), 1.843-22.224; $\mathrm{P}=0.003$; post-HSCT OS: HR, 7.203; 95\% CI, 1.510-34.369; P=0.013; DFS HR, 3.671; 95\% CI, 1.145-11.768; $\mathrm{P}=0.029$ ], post-transplantation minimal residual disease (DFS HR, 4.381; 95\% CI, 1.594-12.040; $\mathrm{P}=0.004$; pre-HSCT OS HR, 11.455; 95\% CI, 1.336-98.179; $\mathrm{P}=0.026$ ) and disease status (OS HR, 0.330; 95\% CI, 0.128-0.848; $\mathrm{P}=0.021$; post-HSCT OS HR, 0.195; 95\% CI, 0.050-0.762; $\mathrm{P}=0.019$; DFS: HR, 0.278; 95\% CI, 0.091-0.851; $\mathrm{P}=0.025$ ) could serve as an adverse prognostic factor in patients with AL treated with allo-HSCT. ${ }^{18} \mathrm{~F}-\mathrm{FDG}$ PET/CT before and/or after allo-HSCT was a predictor for OS and DFS in patients
\end{abstract}

Correspondence to: Dr Shengming Deng, Department of Nuclear Medicine, The First Affiliated Hospital of Soochow University, 188 Shizi Street, Suzhou, Jiangsu 215006, P.R. China

E-mail: dshming@163.com

*Contributed equally

Key words: fluorodeoxy glucose F18, positron emission tomography/CT, acute leukemia, hematopoietic stem cell transplantation with AL. $\mathrm{E}_{\mathrm{N}} \mathrm{E}_{\mathrm{M}} \mathrm{E}_{\mathrm{S}}$ involvement detected using ${ }^{18} \mathrm{~F}-\mathrm{FDG}$ $\mathrm{PET} / \mathrm{CT}$ may help identify patients with $\mathrm{AL}$ who are likely to have unfavorable clinical outcomes.

\section{Introduction}

As a type of hematopoietic system disease, acute leukemia (AL) is characterized by the malignant transformation of hematopoietic stem and precursor cells within the bone marrow (BM) or thymus (1). AL primarily comprises of acute lymphoblastic (ALL), myeloblastic (AML), undifferentiated (AUL) and mixed-lineage leukemia (AMLL) (2). AL has become a global health concern due to its increasing incidence over the past decade (3). The worldwide incidence of AML has gradually increased from $63.84 \times 10^{3}$ cases in 1990 to $119.57 \times 10^{3}$ cases in 2017 , showing an increase of $87.3 \%$ (4). The USA has diagnosed approximately 5,960 new cases of ALL and reported 1,470 ALL-associated mortalities in 2018 (5). To date, allogeneic hematopoietic stem cell transplantation (allo-HSCT) is the only potentially curative treatment for AL (6). However, allo-HSCT is associated with high-risk of non-relapse-associated mortality and disease relapse, which are causes of mortality of patients with $\mathrm{AL}$ who receive allo-HSCT treatment (7). It has been reported that the incidence of relapse and risk of non-relapse mortality of core binding factor AML are 19.8 and $22.5 \%$ for relapse and 20.9 and $23.3 \%$ for non-relapse mortality, respectively (8). Therefore, reliable prognostic factors are necessary to predict patient outcomes at the time of transplantation.

Positron emission tomography (PET)/CT using the radio-labeled glucose analog ${ }^{18} \mathrm{~F}-2$ '-deoxy-2'-fluorodeoxyglucose $\left({ }^{18} \mathrm{~F}-\mathrm{FDG}\right)$ has been widely used for diagnosis, staging and prognosis prediction of malignant disease, including hematological malignancy $(9,10)$. Although a number of studies concerning the prognostic value of ${ }^{18} \mathrm{~F}-\mathrm{FDG} \mathrm{PET} / \mathrm{CT}$ in patients with multiple myeloma or lymphoma undergoing HSCT have been published (11-13), the prognostic value of ${ }^{18} \mathrm{~F}-\mathrm{FDG}$ PET/CT imaging in patients with AL has yet to be established. The present study aimed to determine whether ${ }^{18} \mathrm{~F}-\mathrm{FDG} \mathrm{PET} / \mathrm{CT}$ performed before and/or after allo-HSCT could be used to predict clinical outcomes in AL. 


\section{Materials and methods}

Ethical approval. The present retrospective study (trial registration no. ChiCTR1900024823) was approved by the Ethics Committee of the First Affiliated Hospital of Soochow University (Suzhou, China; approval no. 2019055), with a waiver of informed consent.

Patients. From January 2011 to January 2019, patients with AL who underwent ${ }^{18} \mathrm{~F}-\mathrm{FDG} \mathrm{PET} / \mathrm{CT}$ before or after allo-HSCT were retrospectively enrolled in the present study. The following inclusion criteria were used: Histologically confirmed as AL and patients who underwent ${ }^{18} \mathrm{~F}$-FDG PET/CT before or after allo-HSCT. The following exclusion criteria were used: i) Patients with lymphoma cell leukemia, ii) digital image data unavailable for retrospective analysis, iii) patients who received granulocyte colony-stimulating factor (G-CSF) therapy $<1$ month before PET/CT scan and iv) time interval between day 0 of allo-HSCT and PET/CT scan $>12$ months. Patients were followed-up for $\geq 4$ months after allo-HSCT. Follow-up data were collected through clinic or over the phone, with an average follow-up of 25.2 months.

A total of 96 patients with AL were retrospectively reviewed in the present study. A total of 24 patients were excluded from the final analysis. Among these, 15 patients had lymphoma cell leukemia, three received G-CSF therapy $<1$ month before $\mathrm{PET} / \mathrm{CT}$ scan and six underwent PET/CT scan $>12$ months before or after allo-HSCT. A total of 72 patients [21 female, 51 male; mean age \pm standard deviation $(\mathrm{SD}), 31 \pm 13$ years; range, 7-60 years] were retrospectively enrolled in the final analysis.

Risk stratification of patients with AML was primarily assessed according to European Leukemia Net (ELN) 2017 (14) and risk stratification of patients with ALL was primarily assessed according to the NCCN Guidelines (15). For patients with insufficient information for NCCN 2019 and ELN 2017, risk stratification was independently evaluated by two experienced hematologists, and results were recorded by consensus. According to NCCN 2019 Guidelines, ALL was divided into high-risk and low-risk, while AML was divided into high-risk, intermediate-risk and low-risk according to ELN 2017. Therefore, in order to better analyze risk stratification, low-risk and intermediate-risk patients with AML were combined as low-risk ones.

Image acquisition. ${ }^{18} \mathrm{~F}-\mathrm{FDG}$ PET/CT imaging was performed using a standard whole-body protocol as previously described (15). All patients were fasted for $\geq 6 \mathrm{~h}$ before ${ }^{18} \mathrm{~F}$-FDG $\mathrm{PET} / \mathrm{CT}$ examination. The baseline blood glucose level was $<11 \mathrm{mmol} / 1$. At $60 \mathrm{~min}$ after administration of ${ }^{18} \mathrm{~F}-\mathrm{FDG}$ (dose, $0.12 \mathrm{mCi} / \mathrm{kg}$ ), ${ }^{18} \mathrm{~F}-\mathrm{FDG}$ PET/CT was performed using a Discovery STE PET/CT scanner (General Electric Medical Systems, Milwaukee, WI, USA). Transmission data were acquired via whole-body CT $(140 \mathrm{kV} ; 120 \mathrm{~mA}$; pitch, 1.75; transaxial FOV, $700 \mathrm{~mm}$; slice thickness, $3.75 \mathrm{~mm}$; rotation time, $0.8 \mathrm{sec})$. PET emission data in 3D-mode were analyzed from the vertex of the skull to the proximal thigh, with 2-3 min per bed position. Transverse PET slices were reconstructed using a standard iterative algorithm (ordered-subset expectation maximization), with low-dose CT data utilized for image
Table I. Patient characteristics.

\begin{tabular}{|c|c|}
\hline Characteristics & $\mathrm{N}$ \\
\hline \multicolumn{2}{|l|}{ Age, years } \\
\hline$\leq 20$ & 20 \\
\hline$>20$ & 52 \\
\hline Mean \pm standard deviation (range) ${ }^{\mathrm{a}}$ & $31 \pm 13(7-60)$ \\
\hline \multicolumn{2}{|l|}{ Sex } \\
\hline Female & 21 \\
\hline Male & 51 \\
\hline \multicolumn{2}{|l|}{ Acute leukemia type } \\
\hline Lymphoblastic & 33 \\
\hline Myeloblastic & 36 \\
\hline Mixed-lineage & 3 \\
\hline \multicolumn{2}{|l|}{ De novo or secondary } \\
\hline De novo & 69 \\
\hline Secondary & 3 \\
\hline \multicolumn{2}{|l|}{$\begin{array}{l}\text { Before or after allogeneic hematopoietic } \\
\text { stem cell transplantation }\end{array}$} \\
\hline Before & 19 \\
\hline After & 46 \\
\hline Before and after & 7 \\
\hline Median follow-up & 21 months \\
\hline \multicolumn{2}{|l|}{ Lactate dehydrogenase } \\
\hline High & 42 \\
\hline Normal & 30 \\
\hline \multicolumn{2}{|c|}{ White blood cell count at diagnosis, $\times 10^{9} / 1$} \\
\hline$\leq 20$ & 45 \\
\hline$>20$ & 27 \\
\hline \multicolumn{2}{|l|}{ Risk stratification } \\
\hline Good & 20 \\
\hline Poor & 49 \\
\hline Unknown & 3 \\
\hline \multicolumn{2}{|l|}{ Disease status } \\
\hline Complete remission & 54 \\
\hline Non-remission & 18 \\
\hline \multicolumn{2}{|l|}{ Pre-transplantation MRD } \\
\hline Positive & 43 \\
\hline Negative & 18 \\
\hline \multicolumn{2}{|l|}{ Post-transplantation MRD } \\
\hline Positive & 22 \\
\hline Negative & 20 \\
\hline
\end{tabular}

apresented as mean \pm standard deviation (range). MRD, minimal residual disease.

fusion and attenuation correction, using the Xeleris workstation software (GE Healthcare; ADW4.1).

Image analysis. ${ }^{18} \mathrm{~F}-\mathrm{FDG} \mathrm{PET} / \mathrm{CT}$ images were independently evaluated by two experienced nuclear medicine physicians who were blinded to the clinical information of all subjects, and the results were recorded by consensus. Increased ${ }^{18} \mathrm{~F}-\mathrm{FDG}$ uptake in the BM and spleen was defined as diffuse 
Table II. Univariate and multivariate analysis of OS of all PET/CT examinations.

A, Univariate analysis

\begin{tabular}{|c|c|c|}
\hline Parameters & P-value & Hazard ratio $(95 \% \mathrm{CI})$ \\
\hline Sex, Male vs. Female & 0.8524 & $0.9200(0.3840-2.2070)$ \\
\hline Age, $>21$ years & 0.5771 & $1.3620(0.4600-4.0390)$ \\
\hline Risk stratification & 0.1642 & $1.8720(0.7740-4.5270)$ \\
\hline White blood cell count, $>20 \times 10^{9} / 1$ & 0.0772 & $2.1740(0.9190-5.1420)$ \\
\hline Elevated lactate dehydrogenase, $>245 \mathrm{U} / 1$ & 0.5077 & $0.7590(0.3350-1.7170)$ \\
\hline Acute leukemia type & 0.1690 & $1.8250(0.7740-4.3000)$ \\
\hline PET-positive & 0.1234 & $2.0900(0.8182-5.3370)$ \\
\hline Increased $\mathrm{BM}{ }^{18} \mathrm{~F}-\mathrm{FDG}$ uptake & 0.4406 & $0.6850(0.2620-1.7910)$ \\
\hline Focal BM ${ }^{18}$ F-FDG uptake & 0.3840 & $1.2950(0.0360-4.3590)$ \\
\hline Increased splenic ${ }^{18} \mathrm{~F}$-FDG uptake & 0.6955 & $0.8480(0.3720-1.9340)$ \\
\hline${ }^{18} \mathrm{~F}$-FDG-avid lymph nodes $>1.5 \mathrm{~cm}$ & 0.0140 & $2.8710(1.0950-7.5290)$ \\
\hline $\mathrm{E}_{\mathrm{N}} \mathrm{E}_{\mathrm{M}} \mathrm{E}_{\mathrm{S}}$ involvement & 0.0003 & $4.5450(2.0080-10.2900)$ \\
\hline Disease status & 0.0009 & $0.1930(0.0726-0.5100)$ \\
\hline Pre-transplantation MRD & 0.1737 & $2.1150(0.7188-6.2240)$ \\
\hline Post-transplantation MRD & $0.0017^{\mathrm{a}}$ & $4.5940(1.7740-11.9000)$ \\
\hline
\end{tabular}

B, Multivariate analysis

\begin{tabular}{lcc}
\hline Parameters & P-value & Hazard ratio (95\% CI) \\
\hline $\mathrm{E}_{\mathrm{N}} \mathrm{E}_{\mathrm{M}} \mathrm{E}_{\mathrm{S}}$ involvement & 0.0030 & $6.3990(1.8430-22.2240)$ \\
${ }^{18} \mathrm{~F}-\mathrm{FDG}$-avid lymph nodes $>1.5 \mathrm{~cm}$ & 0.6620 & - \\
Disease status & 0.0210 & $0.3300(0.1280-0.8480)$ \\
Post-transplantation MRD & 0.1180 & - \\
\hline
\end{tabular}

PET, positron emission tomography; BM: Bone marrow; ${ }^{18} \mathrm{~F}-\mathrm{FDG},{ }^{18} \mathrm{~F}-2$ '-deoxy-2'-fluorodeoxyglucose; $\mathrm{E}_{\mathrm{N}} \mathrm{E}_{\mathrm{M}} \mathrm{E}_{\mathrm{S}}$, extranodal, extramedullary and extrasplenic; MRD, minimal residual disease.

and/or focal ${ }^{18} \mathrm{~F}$-FDG uptake $\geq$ normal liver $(16,17)$. The region of interest located in the right lobe of the liver served as the reference. Diffuse or focal ${ }^{18} \mathrm{~F}$-FDG uptake above the mediastinal blood pool or background uptake excluding infectious, inflammatory or other neoplastic diseases was considered as other PET-positive (18).

Statistical analysis. GraphPad Prism (version 5.0; GraphPad Software, Inc.) and SPSS software (version 19.0; IBM, Corp.) were used for statistical analysis. In order to assess the prognostic value of ${ }^{18} \mathrm{~F}$-FDG PET/CT imaging, overall survival (OS) and disease-free survival (DFS) were selected as the endpoints. OS was defined as the period from the date of allo-HSCT to the date of death. DFS was defined as the period from the date of allo-HSCT to the date of relapse or death. OS and DFS were estimated via the Kaplan-Meier method, and differences among groups were evaluated by a log-rank test. The relation between factors associated with OS and DFS was estimated using the Kaplan-Meier method, and the log-rank test was used for univariate analysis. For multivariate analysis, risk factors with statistical significance upon univariate analysis were introduced into a Cox proportional hazards model. $\mathrm{P}<0.05$ was considered to indicate a statistically significant difference.

\section{Results}

Characteristics of patients. A total of 72 patients (21 females, 51 males; mean age \pm SD, $31 \pm 13$ years; range, 7-60 years) were enrolled between January 2011 and January 2019. Of these 72 patients, 33 had ALL, 36 had AML and three had AMLL. In addition, 19 patients underwent ${ }^{18} \mathrm{~F}$-FDG PET/CT before allo-HSCT, 46 underwent ${ }^{18} \mathrm{~F}$-FDG PET/CT after allo-HSCT and seven underwent ${ }^{18} \mathrm{~F}$-FDG PET/CT both before and after allo-HSCT. Therefore, 79 examinations of 72 patients were enrolled in the study. The median follow-up was 21 months. The white blood cell count at diagnosis was $>20 \times 10^{9} / 1$ in 27 patients. The lactate dehydrogenase levels ( $>245 \mathrm{U} / \mathrm{L})$ were high in 42 patients (19). Detailed characteristics of patients are presented in Table I.

PET/CT results. A total of 63 examinations were ${ }^{18} \mathrm{~F}-\mathrm{FDG}$ PET positive, whereas 16 examinations were ${ }^{18} \mathrm{~F}$-FDG PET negative. Increased $\mathrm{BM}{ }^{18} \mathrm{~F}-\mathrm{FDG}$ uptake was observed in $24 \%$ (19/79) of examinations, including a homogeneous/diffuse pattern throughout the body in 13 examinations and an inhomogeneous/focal pattern or co-existing inhomogeneous/focal and homogeneous/diffuse patterns in six examinations. 
Table III. Univariate and multivariate analysis of disease-free survival of all PET/CT examinations.

\begin{tabular}{lcc} 
A, Univariate analysis & & \\
\hline Parameters & P-value & Hazard ratio (95\% CI) \\
\hline Sex, Male vs. Female & 0.8031 & $0.900(0.398-2.038)$ \\
Age, $>21$ years & 0.5537 & $1.339(0.510-3.520)$ \\
Risk stratification & 0.9173 & $0.958(0.423-2.166)$ \\
White blood cell count, $>20 x 10^{9} / 1$ & 0.4990 & $1.307(0.602-2.840)$ \\
Elevated lactate dehydrogenase, $>245$ U/1 & 0.2326 & $0.632(0.297-1.343)$ \\
Acute leukemia type & 0.3659 & $1.426(0.661-3.078)$ \\
PET-positive & 0.2950 & $1.621(0.656-4.004)$ \\
Increased BM ${ }^{18}$ F-FDG uptake & 0.8546 & $0.920(0.376-2.249)$ \\
Focal BM ${ }^{18}$ F-FDG uptake & 0.5620 & $1.567(0.470-5.226)$ \\
Increased splenic ${ }^{18}$ F-FDG uptake & 0.2911 & $0.661(0.306-1.426)$ \\
${ }^{18}$ F-FDG-avid lymph nodes $>1.5 \mathrm{~cm}$ & 0.0080 & $3.743(1.515-9.245)$ \\
$\mathrm{E}_{\mathrm{N}} \mathrm{E}_{\mathrm{M}} \mathrm{E}_{\mathrm{S}}$ involvement & 0.0005 & $3.793(1.782-8.071)$ \\
Disease status & 0.0091 & $0.285(0.111-0.732)$ \\
Pre-transplantation MRD & 0.3819 & $1.489(0.610-3.634)$ \\
Post-transplantation MRD & 0.0016 & $4.171(1.722-10.100)$
\end{tabular}

B, Multivariate analysis

\begin{tabular}{lcc}
\hline Parameters & P-value & Hazard ratio (95\% CI) \\
\hline $\mathrm{E}_{\mathrm{N}} \mathrm{E}_{\mathrm{M}} \mathrm{E}_{\mathrm{S}}$ involvement & 0.0950 & - \\
${ }^{18} \mathrm{~F}-\mathrm{FDG}$-avid lymph nodes $>1.5 \mathrm{~cm}$ & 0.2190 & - \\
Disease status & 0.2340 & - \\
Post-transplantation MRD & $0.0040^{\mathrm{a}}$ & $4.381(1.594-12.040)$ \\
\hline
\end{tabular}

${ }^{\mathrm{a}} \mathrm{P}<0.05$. PET, positron emission tomography; BM, bone marrow; 18F-FDG, 18F-2'-deoxy-2'-fluorodeoxyglucose; ENEMES: Extranodal, extramedullary and extrasplenic; MRD, minimal residual disease.

Increased splenic ${ }^{18}$ F-FDG uptake was detected in $14 \%(11 / 79)$ of examinations, including a homogeneous/diffuse pattern in 10 examinations and an inhomogeneous/focal pattern in one examination. ${ }^{18} \mathrm{~F}-\mathrm{FDG}$-avid lymph nodes were observed in $38 \%(30 / 79)$ of examinations, including lymph nodes $>1.5 \mathrm{~cm}$ in eight examinations and lymph nodes $\leq 1.5 \mathrm{~cm}$ in 22 examinations. One patient with lymph nodes $<1.5 \mathrm{~cm}$ in short axis demonstrated inflammation.

$\mathrm{E}_{\mathrm{N}} \mathrm{E}_{\mathrm{M}} \mathrm{E}_{\mathrm{S}}$ involvement was detected by PET/CT in $44 \%$ $(35 / 79)$ of examinations. The most common ${ }^{18} \mathrm{~F}$-FDG-avid site was bone [16\% (13/79) of examinations]. Other ${ }^{18} \mathrm{~F}-\mathrm{FDG}$-avid sites included nasopharynx (eight, including two inflammations), soft tissue (seven), lung (five, including three inflammations), breast (four), testes (three), brain (three, including one abscess), kidney, heart, skin, pleura, parotid gland, liver (two each), adrenal, uterus, eyelid and submandibular gland (one examination each).

Prognosis prediction of all PET/CT examinations. Univariate and multivariate Cox regression analysis of all 79 examinations was used to assess the effect of ${ }^{18} \mathrm{~F}-\mathrm{FDG}$ PET/CT variables and clinical parameters on OS and DFS (Tables II and III). In univariate analysis, ${ }^{18} \mathrm{~F}-\mathrm{FDG}$-avid lymph nodes $>1.5 \mathrm{~cm}$, $\mathrm{E}_{\mathrm{N}} \mathrm{E}_{\mathrm{M}} \mathrm{E}_{\mathrm{S}}$ involvement, disease status and post-transplantation minimal residual disease (MRD) were significantly associated with OS and DFS. However, only $\mathrm{E}_{\mathrm{N}} \mathrm{E}_{\mathrm{M}} \mathrm{E}_{\mathrm{S}}$ involvement [hazard ratio (HR), 6.399; 95\% $\mathrm{CI}, 1.843-22.224 ; \mathrm{P}=0.003$ ] and disease status (HR, 0.330; 95\% CI, 0.128-0.848; $\mathrm{P}=0.021)$ were significantly associated with OS in multivariate analysis and only post-transplantation MRD was significantly associated with DFS (HR, 4.381; 95\% CI, 1.594-12.040; $\mathrm{P}=0.004$ ).

Kaplan-Meier analysis for OS of all 79 examinations showed that patients with negative $E_{N} E_{M} E_{S}$ involvement and complete remission (CR) had a better OS compared with patients with non-remission (NR) and positive $E_{N} E_{M} E_{S}$ involvement (Fig. 1A and $\mathrm{C}$, respectively). The median OS was 28 months in patients with $\mathrm{E}_{\mathrm{N}} \mathrm{E}_{\mathrm{M}} \mathrm{E}_{\mathrm{S}}$ involvement; patients without $\mathrm{E}_{\mathrm{N}} \mathrm{E}_{\mathrm{M}} \mathrm{E}_{\mathrm{S}}$ involvement did not reach the median OS $(\mathrm{P}<0.001)$. The median OS was 24 months in patients with $\mathrm{CR}$; patients with NR did not reach the median OS $(\mathrm{P}<0.001)$. Kaplan-Meier analysis demonstrated that patients with positive $E_{N} E_{M} E_{S}$ involvement and $N R$ exhibited a shorter DFS compared with patients with negative $\mathrm{E}_{\mathrm{N}} \mathrm{E}_{\mathrm{M}} \mathrm{E}_{\mathrm{S}}$ involvement and CR (Fig. 1B and D, respectively). Kaplan-Meier analysis for OS showed that the patients with positive post-transplantation MRD had a shorter OS (median OS, 24 months) compared with patients in the negative post-transplantation MRD group (median OS, not reached; $\mathrm{P}<0.01$; Fig. 1E). Kaplan-Meier 
Table IV. Univariate and multivariate analysis of OS after allo-HSCT.

A, Univariate analysis.

\begin{tabular}{lcc}
\hline Parameters & P-value & Hazard ratio (95\% CI) \\
\hline Sex, Male vs. Female & 0.4427 & $1.48400(0.54190-4.06200)$ \\
Age, $>21$ years & 0.9861 & $1.013000(0.22840-4.49700)$ \\
Risk stratification & 0.1832 & $1.95600(0.72830-5.25600)$ \\
White blood cell count, $>20 x 10^{9} / 1$ & 0.1070 & $3.33000(1.24000-8.94500)$ \\
Elevated lactate dehydrogenase, $>245 \mathrm{U} / 1$ & 0.6205 & $0.79020(0.31100-2.00700)$ \\
Acute leukemia type & 0.3711 & $0.65000(0.25300-1.67100)$ \\
Increased bone marrow ${ }^{18}$ F-FDG uptake & 0.8303 & $0.81620(0.12750-5.22700)$ \\
Increased splenic ${ }^{18}$ F-FDG uptake & 0.7893 & $0.87170(0.31830-2.38700)$ \\
${ }^{18}$ F-FDG-avid lymph nodes $>1.5 \mathrm{~cm}$ & $0.0100^{\mathrm{a}}$ & $0.11100(0.02100-0.57500)$ \\
$\mathrm{E}_{\mathrm{N}} \mathrm{E}_{\mathrm{M}} \mathrm{E}_{\mathrm{S}}$ involvement & 0.0044 & $3.87800(1.52600-9.85400)$ \\
Disease status & $0.0080^{\mathrm{a}}$ & $0.20440(0.06316-0.66130)$ \\
Pre-transplantation MRD & 0.0743 & $2.83200(0.90290-8.88200)$ \\
Post-transplantation MRD & 0.0463 & $3.24300(1.01900-10.32000)$ \\
\hline
\end{tabular}

B, Multivariate analysis

\begin{tabular}{lcc}
\hline Parameters & P-value & Hazard ratio (95\% CI) \\
\hline $\mathrm{E}_{\mathrm{N}} \mathrm{E}_{\mathrm{M}} \mathrm{E}_{\mathrm{S}}$ involvement & 0.0130 & $7.20300(1.51000-34.36900)$ \\
${ }^{18} \mathrm{~F}-\mathrm{FDG}$-avid lymph nodes $>1.5 \mathrm{~cm}$ & 0.8920 & - \\
Disease status & 0.0190 & $0.19500(0.05000-0.76200)$
\end{tabular}

${ }^{\mathrm{a}} \mathrm{P}<0.05$. MRD, minimal residual disease; PET: Positron emission tomography; ${ }^{18} \mathrm{~F}-\mathrm{FDG}$ : ${ }^{18} \mathrm{~F}-2$ '-deoxy-2'-fluorodeoxyglucose; $\mathrm{E}_{\mathrm{N}} \mathrm{E}_{\mathrm{M}} \mathrm{E}_{\mathrm{S}}$ : Extranodal, extramedullary and extrasplenic.
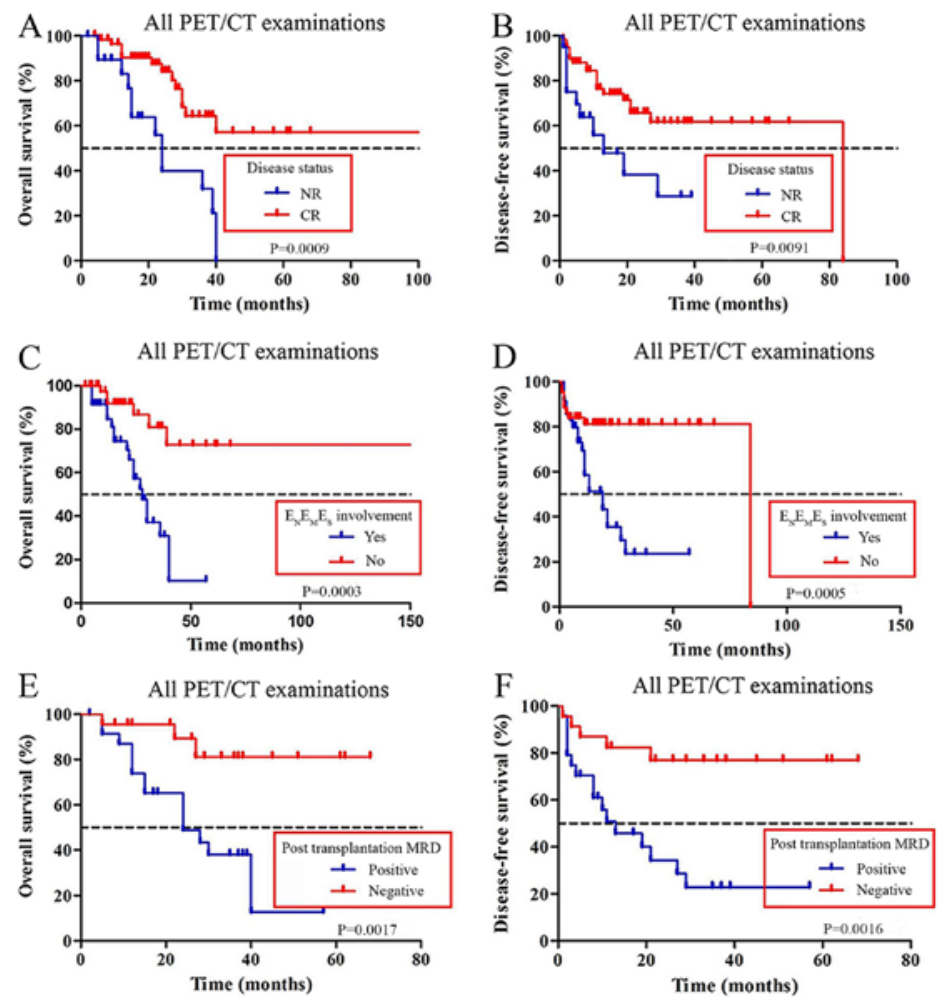

Figure 1. Kaplan-Meier analysis for overall survival and disease-free survival according to (A and B) disease status, (C and D) presence of $E_{N} E_{M} E_{S}$ involvement and (E and $\mathrm{F}$ ) post-transplant MRD in patients with $\mathrm{AL}$. The dotted line represents $50 \%$ survival rate. $\mathrm{E}_{\mathrm{N}} \mathrm{E}_{\mathrm{M}} \mathrm{E}_{\mathrm{S}}$, extranodal, extramedullary and extrasplenic; $\mathrm{MRD}$, minimal residual disease; PET, positron emission tomography; NR, non-remission; CR, complete remission. 
Table V. Univariate and multivariate analysis of disease-free survival after allo-HSCT.

A, Univariate analysis

\begin{tabular}{|c|c|c|}
\hline Parameters & P-value & Hazard ratio $(95 \% \mathrm{CI})$ \\
\hline Sex, Male vs. Female & 0.2981 & $1.6600(0.6391-4.3110)$ \\
\hline Age, $>21$ years & 0.9243 & $0.9405(0.2654-3.3320)$ \\
\hline Risk stratification & 0.8912 & $1.0650(0.4308-2.6340)$ \\
\hline White blood cell count, $>20 \times 10^{9} / 1$ & 0.3043 & $1.5590(0.6680-3.6400)$ \\
\hline Elevated lactate dehydrogenase, $>245 \mathrm{U} / 1$ & 0.1586 & $0.5393(0.2286-1.2720)$ \\
\hline Acute leukemia type & 0.4395 & $0.7129(0.3023-1.6810)$ \\
\hline Increased bone marrow ${ }^{18} \mathrm{~F}-\mathrm{FDG}$ uptake & 0.2394 & $3.6020(0.4259-30.4600)$ \\
\hline Increased splenic ${ }^{18} \mathrm{~F}-\mathrm{FDG}$ uptake & 0.4104 & $0.6315(0.2114-1.8870)$ \\
\hline${ }^{18} \mathrm{~F}$-FDG-avid lymph nodes $>1.5 \mathrm{~cm}$ & 0.0143 & $0.1980(0.0560-0.7060)$ \\
\hline $\mathrm{E}_{\mathrm{N}} \mathrm{E}_{\mathrm{M}} \mathrm{E}_{\mathrm{S}}$ involvement & 0.0002 & $5.03700(2.1550-11.7700)$ \\
\hline Disease status & 0.0467 & $0.3375(0.1157-0.9842)$ \\
\hline Pre-transplantation MRD & 0.5118 & $1.4060(0.5081-3.8890)$ \\
\hline Post-transplantation MRD & 0.0335 & $3.0720(1.0920-8.6460)$ \\
\hline \multicolumn{3}{|l|}{$\mathrm{B}$, Multivariate analysis } \\
\hline Parameters & P-value & Hazard ratio $(95 \% \mathrm{CI})$ \\
\hline $\mathrm{E}_{\mathrm{N}} \mathrm{E}_{\mathrm{M}} \mathrm{E}_{\mathrm{S}}$ involvement & 0.0290 & $3.6710(1.1450-11.7680)$ \\
\hline${ }^{18} \mathrm{~F}$-FDG-avid lymph nodes $>1.5 \mathrm{~cm}$ & 0.0630 & - \\
\hline Disease status & 0.0250 & $0.2780(0.0910-0.8510)$ \\
\hline
\end{tabular}

MRD, minimal residual disease; PET, positron emission tomography; ${ }^{18} \mathrm{~F}-\mathrm{FDG},{ }^{18} \mathrm{~F}-2$ '-deoxy-2'-fluorodeoxyglucose; $\mathrm{E}_{\mathrm{N}} \mathrm{E}_{\mathrm{M}} \mathrm{E}_{\mathrm{S}}$, extranodal, extramedullary and extrasplenic.

Table VI. Univariate and multivariate analysis of OS before allo-HSCT.

A, Univariate analysis

\begin{tabular}{llc}
\hline Parameters & P-value & Hazard ratio (95\% CI) \\
\hline Sex, Male vs. Female & 0.08140 & $0.21240(0.037180-1.21300)$ \\
Age, $>21$ years & 0.54090 & $1.77200(0.28310-11.10000)$ \\
Risk stratification & 0.62150 & $1.71400(0.25320-11.61000)$ \\
White blood cell count, $>20 x 10^{9} / 1$ & 0.72150 & $0.69950(0.09806-4.99100)$ \\
Elevated lactate dehydrogenase, $>245 \mathrm{U} / 1$ & 0.86300 & $0.86450(0.16540-4.51900)$ \\
Acute leukemia type & 0.33770 & $3.16700(0.29990-33.45000)$ \\
Increased bone marrow ${ }^{18}$ F-FDG uptake & 0.45810 & $0.52100(0.09307-2.91600)$ \\
Increased splenic ${ }^{18}$ F-FDG uptake & 0.90260 & $1.12300(0.17450-7.23000)$ \\
${ }^{18}$ F-FDG-avid lymph nodes $>1.5 \mathrm{~cm}$ & 0.77450 & $0.31300(0.01500-6.40900)$ \\
$\mathrm{E}_{\mathrm{N}} \mathrm{E}_{\mathrm{M}} \mathrm{E}_{\mathrm{S}}$ involvement & $0.03320^{\mathrm{a}}$ & $6.16500(1.15600-32.88000)$ \\
Disease status & $0.02170^{\mathrm{a}}$ & $0.11280(0.01750-0.72740)$ \\
Pre-transplantation MRD & 0.62630 & $0.50040(0.03083-8.12000)$ \\
Post-transplantation MRD & $0.00360^{\mathrm{a}}$ & $14.58000(2.40600-88.37000)$ \\
\hline
\end{tabular}

$\mathrm{B}$, Multivariate analysis

\begin{tabular}{llc}
\hline Parameters & P-value & Hazard ratio (95\% CI) \\
\hline $\mathrm{E}_{\mathrm{N}} \mathrm{E}_{\mathrm{M}} \mathrm{E}_{\mathrm{S}}$ involvement & 0.32900 & - \\
Disease status & 0.69100 & - \\
Post-transplantation MRD & $0.02600^{\mathrm{a}}$ & $11.45500(1.33600-98.17900)$ \\
\hline
\end{tabular}

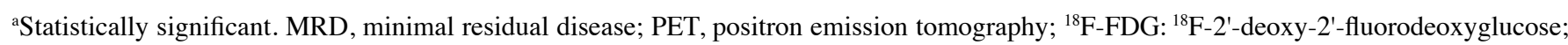
$\mathrm{E}_{\mathrm{N}} \mathrm{E}_{\mathrm{M}} \mathrm{E}_{\mathrm{S}}$ : Extranodal, extramedullary and extrasplenic. 
Table VII. Univariate analysis of disease-free survival before allo-HSCT.

\begin{tabular}{lcr}
\hline Parameters & P-value & Hazard ratio (95\% CI) \\
\hline Sex, Male vs. Female & 0.3185 & $0.42490(0.07905-2.28400)$ \\
Age, $>21$ years & 0.4728 & $1.90700(0.32730-11.11000)$ \\
Risk stratification & 0.7556 & $0.75070(0.12340-4.56700)$ \\
White blood cell count, $>20 x 10^{9} / 1$ & 0.1786 & $0.26510(0.03831-1.83500)$ \\
Elevated lactate dehydrogenase, $>245 \mathrm{U} / 1$ & 0.8126 & $0.82120(0.16100-4.18700)$ \\
Acute leukemia type & 0.9503 & $1.06000(0.17150-6.54600)$ \\
Increased BM ${ }^{18}$ F-FDG uptake & 0.8113 & $1.22500(0.23190-6.46900)$ \\
Increased splenic ${ }^{18}$ F-FDG uptake & 0.8397 & $0.84530(0.16590-4.30600)$ \\
${ }^{18}$ F-FDG-avid lymph nodes $>1.5 \mathrm{~cm}$ & 0.1130 & $0.17000(0.01400-2.09100)$ \\
$\mathrm{E}_{\mathrm{N}} \mathrm{E}_{\mathrm{M}} \mathrm{E}_{\mathrm{S}}$ involvement & 0.6853 & $1.40400(0.27180-7.25700)$ \\
Disease status & 0.0633 & $0.14470(0.01882-1.11300)$ \\
Pre-transplantation MRD & 0.6146 & $1.63900(0.23960-11.21000)$ \\
Post-transplantation MRD & 0.0065 & $15.91000(2.17100-116.60000)$
\end{tabular}

MRD, minimal residual disease; BM, bone marrow; ${ }^{18} \mathrm{~F}-\mathrm{FDG},{ }^{18} \mathrm{~F}-2$ '-deoxy-2'-fluorodeoxyglucose; $\mathrm{E}_{\mathrm{N}} \mathrm{E}_{\mathrm{M}} \mathrm{E}_{\mathrm{S}}$, extranodal, extramedullary and extrasplenic.

A

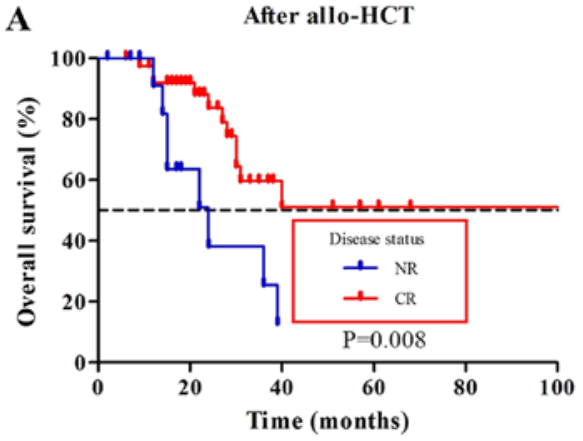

C

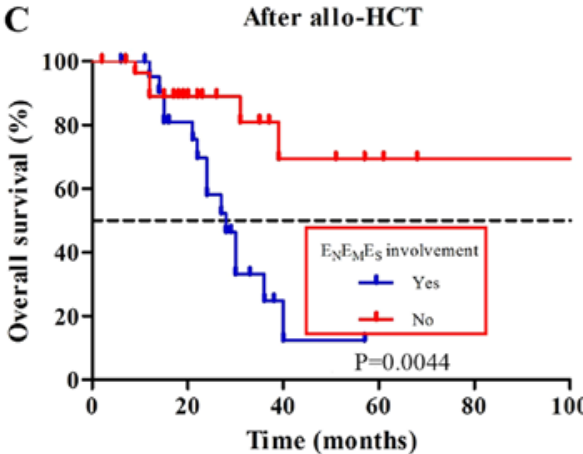

B

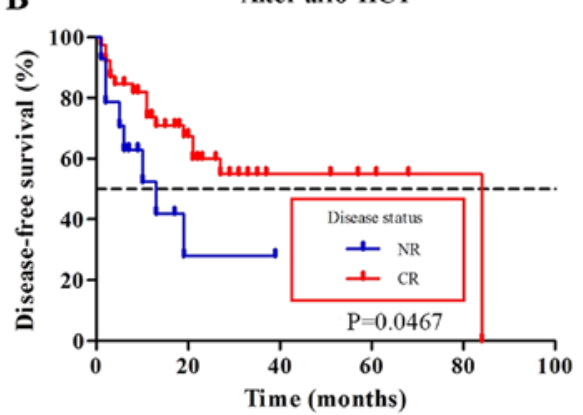

D

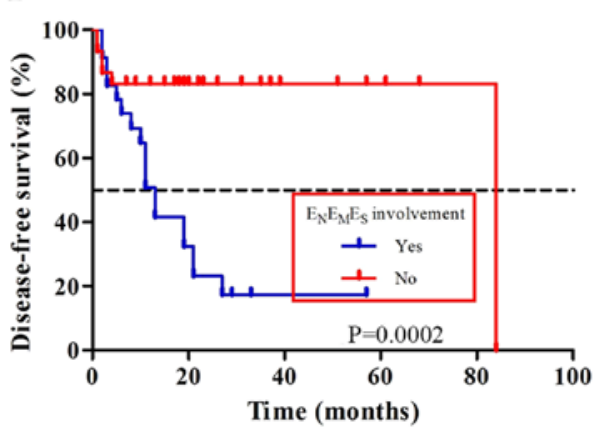

Figure 2. Kaplan-Meier analysis for overall survival and disease-free survival of patients with acute leukemia according to (A and B) disease status and $\left(\mathrm{C}\right.$ and D) presence of $\mathrm{E}_{\mathrm{N}} \mathrm{E}_{\mathrm{M}} \mathrm{E}_{\mathrm{S}}$ involvement detected via ${ }^{18} \mathrm{~F}-2$ '-deoxy-2'-fluorodeoxyglucose positron emission tomography/CT scan after allo-HSCT. The dotted line represents $50 \%$ survival rate. $\mathrm{E}_{\mathrm{N}} \mathrm{E}_{\mathrm{M}} \mathrm{E}_{\mathrm{S}}$, extranodal, extramedullary and extrasplenic; allo-HSCT, allogeneic hematopoietic stem cell transplantation; NR, non-remission; $\mathrm{CR}$, complete remission.

analysis for DFS showed that patients with positive post-transplantation MRD had a shorter DFS (median DFS, 29 months) compared with the patients in the negative post-transplantation MRD group (median DFS, not reached; $\mathrm{P}<0.01$; Fig. 1F).

Prognosis prediction of examinations after or before allo-HSCT. For 53 examinations after allo-HSCT, ${ }^{18} \mathrm{~F}-\mathrm{FDG}$-avid lymph nodes $>1.5 \mathrm{~cm}(\mathrm{OS}, \mathrm{P}=0.010$; DFS, $\mathrm{P}=0.014), \mathrm{E}_{\mathrm{N}} \mathrm{E}_{\mathrm{M}} \mathrm{E}_{\mathrm{S}}$ involvement (OS, $\mathrm{P}=0.0044$; DFS, $\mathrm{P}=0.0002$ ) (Fig. 2C and $\mathrm{D}$, respectively), disease status ( $\mathrm{OS}, \mathrm{P}=0.008$; $\mathrm{DFS}, \mathrm{P}=0.467$ ) (Fig. 2A and $\mathrm{B}$ respectively) and post-transplantation MRD (OS, $\mathrm{P}=0.046$; DFS, $\mathrm{P}=0.034$ ) were all univariately associated with OS and DFS (Tables IV and V). In multivariate analysis, $\mathrm{E}_{\mathrm{N}} \mathrm{E}_{\mathrm{M}} \mathrm{E}_{\mathrm{S}}$ involvement (OS: HR, 7.203; 95\% CI, 1.510-34.369; $\mathrm{P}=0.013$; DFS: HR, 3.671; 95\% CI; 1.145-11.768; $\mathrm{P}=0.029)$ and disease status (OS: HR, 0.195; 95\% CI, 0.050-0.762; $\mathrm{P}=0.019$; 

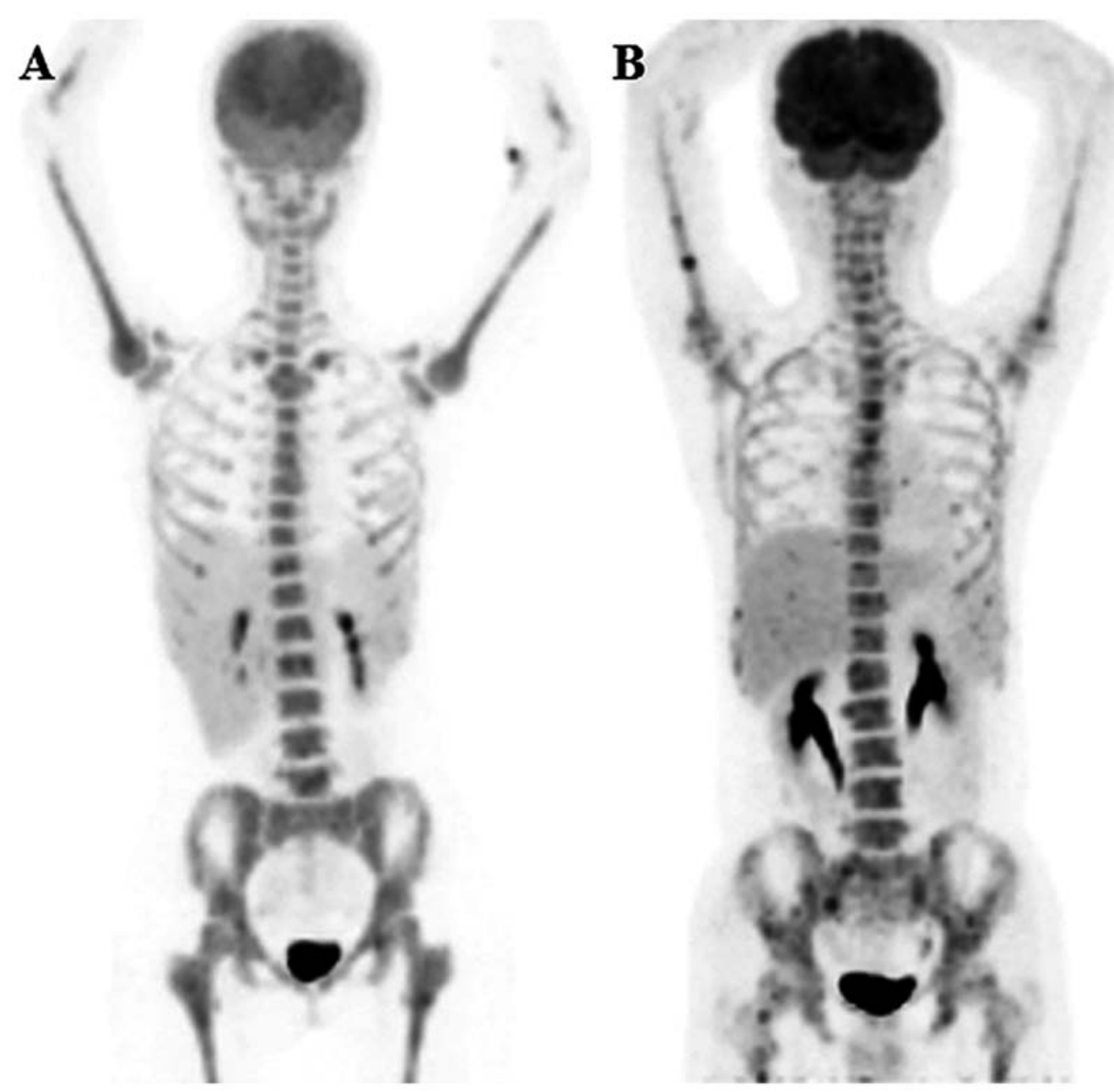

Figure 3. ${ }^{18} \mathrm{~F}-2$-deoxy-2'-fluorodeoxyglucose PET/CT images of a (A) 15-year-old girl and (B) 20-year-old man with acute leukemia prior to allogeneic hematopoietic stem cell transplantation. Maximum-intensity projection PET images show diffuse homogeneous BM uptake and co-existence of focal and diffuse BM uptake, respectively. Both patients were alive at the time of the present study with a follow-up time of 11 and 62 months, respectively. PET, positron emission tomography; BM, bone marrow.

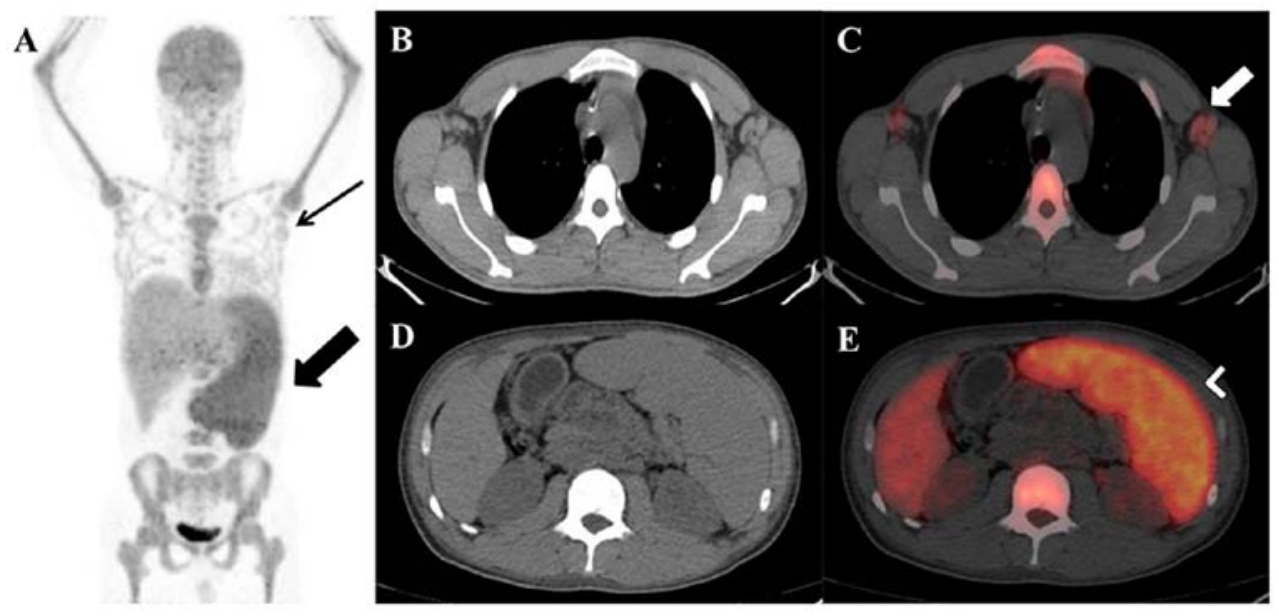

Figure 4. ${ }^{18} \mathrm{~F}-\mathrm{FDG}$ PET/CT imaging in a 29-year-old man with ALL prior to allo-HSCT. (A) Maximum intensity projection PET image showed increased ${ }^{18} \mathrm{~F}$-FDG uptake in splenic parenchyma (thick black arrow), lymph nodes (thin black arrow) and BM in a 29-year-old man with acute lymphoblastic leukemia prior to allo-HSCT. (B-E) Axial PET/CT image revealed splenomegaly (arrowhead) and enlarged axillary lymph nodes (small bold white arrow) of increased ${ }^{18} \mathrm{~F}$-FDG uptake. The patient was alive at the time of the present study, with a follow-up time of 9 months. PET, positron emission tomography; ${ }^{18} \mathrm{~F}-\mathrm{FDG}$, ${ }^{18} \mathrm{~F}-2$ '-deoxy-2'-fluorodeoxyglucose.

DFS: HR, 0.278; 95\% CI, 0.091-0.851; $\mathrm{P}=0.025)$ were significantly associated with OS and DFS (Tables IV and V). For 26 examinations before allo-HSCT, univariate analysis showed that $\mathrm{E}_{\mathrm{N}} \mathrm{E}_{\mathrm{M}} \mathrm{E}_{\mathrm{S}}$ involvement $(\mathrm{P}=0.0332)$, post-transplantation 

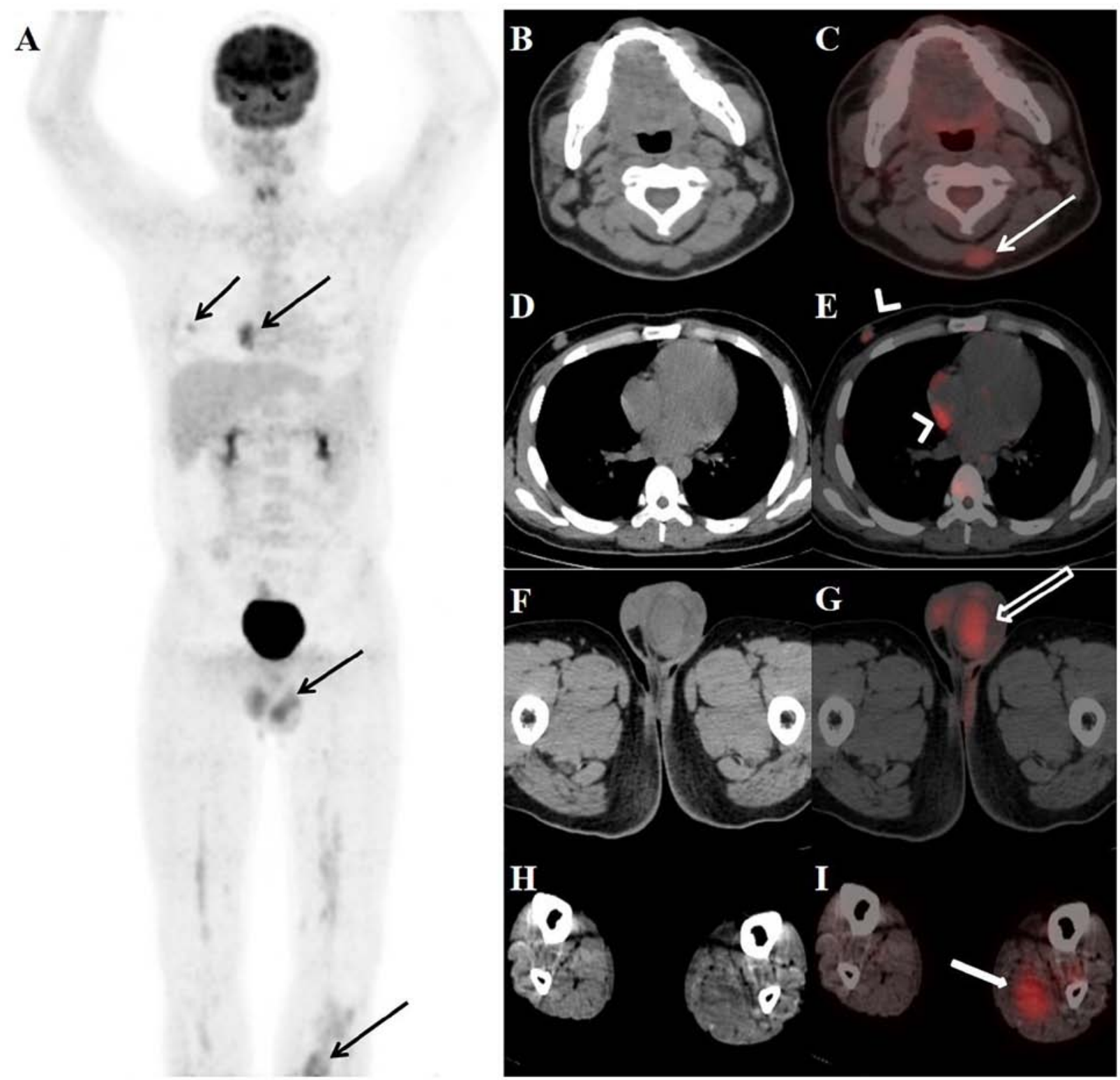

Figure 5. ${ }^{18} \mathrm{~F}-\mathrm{FDG}$ PET/CT imaging in a 22-year-old man with AML after allo-HSCT. (A) Maximum intensity projection PET image showed increased ${ }^{18} \mathrm{~F}-2$-deoxy-2'-fluorodeoxyglucose uptake in $\mathrm{E}_{\mathrm{N}} \mathrm{E}_{\mathrm{M}} \mathrm{E}_{\mathrm{S}}$ sites (black arrow) in a 22-year-old man with acute myeloblastic leukemia after allogeneic human stem cell transplantation. Transverse CT and fusion images showed increased metabolic activity in (B and C) subcutaneous nodules (thin white arrow), (D and E) cardiac region (arrow heads), ( $\mathrm{F}$ and $\mathrm{G}$ ) testes (hollow bold white arrow) and ( $\mathrm{H}$ and $\mathrm{I}$ ) muscles (long bold white arrow). The patient died at 15 months after PET/CT scan. PET, positron emission tomography; $\mathrm{E}_{\mathrm{N}} \mathrm{E}_{\mathrm{M}} \mathrm{E}_{\mathrm{S}}$ extranodal, extramedullary and extrasplenic.

MRD ( $\mathrm{P}=0.0036)$ and disease status $(\mathrm{P}=0.0217)$ were significantly associated with OS (Table VI). Multivariate Cox regression analysis showed that only post-transplantation MRD was significantly associated with OS (HR, 11.455; 95\% CI, 1.336-98.179; $\mathrm{P}=0.026$ ) (Table VI). Post-transplantation MRD was significantly associated with DFS $(\mathrm{P}=0.0065)$ (Table VII).

An example of diffuse homogeneous BM uptake and co-existence of focal and diffuse BM uptake is shown in Fig. 3. Fig. 4 presents an example of ${ }^{18} \mathrm{~F}$-FDG PET/CT examinations with splenic and lymph node uptake. Figs. 5 and 6 present examples of ${ }^{18} \mathrm{~F}$-FDG PET/CT examinations with $\mathrm{E}_{\mathrm{N}} \mathrm{E}_{\mathrm{M}} \mathrm{E}_{\mathrm{S}}$ site uptake.

\section{Discussion}

${ }^{18} \mathrm{~F}-\mathrm{FDG}$ PET/CT is not regularly used in the assessment of leukemia (20). However, a number of clinical studies and case reports have demonstrated the potential of ${ }^{18} \mathrm{~F}-\mathrm{FDG}$ PET/CT in the diagnosis of leukemic bone marrow infiltration and extramedullary disease (EMD), evaluation of granulocytic sarcoma, detection of Richter's syndrome and assessment of graft vs. host disease (21-23). The present study aimed to investigate the prognostic value of ${ }^{18} \mathrm{~F}-\mathrm{FDG} \mathrm{PET} / \mathrm{CT}$ in patients with $\mathrm{AL}$ treated with allo-HSCT. Although increased ${ }^{18} \mathrm{~F}$-FDG uptake by $\mathrm{BM}$, spleen and lymph nodes was observed in patients with $\mathrm{AL}$, none of these factors were independent predictors of OS. Only $E_{N} E_{M} E_{S}$ involvement was significantly associated with OS.

Since AL is a hematological malignancy that originates from BM, increased BM uptake of ${ }^{18} \mathrm{~F}-\mathrm{FDG}$ can be observed in patients with AL (24). However, increased BM ${ }^{18} \mathrm{~F}-\mathrm{FDG}$ uptake can also be observed in benign etiologies and other types of malignant infiltration $(25,26)$. Jeong et al (27), performed a meta-analysis to evaluate the prognostic value 

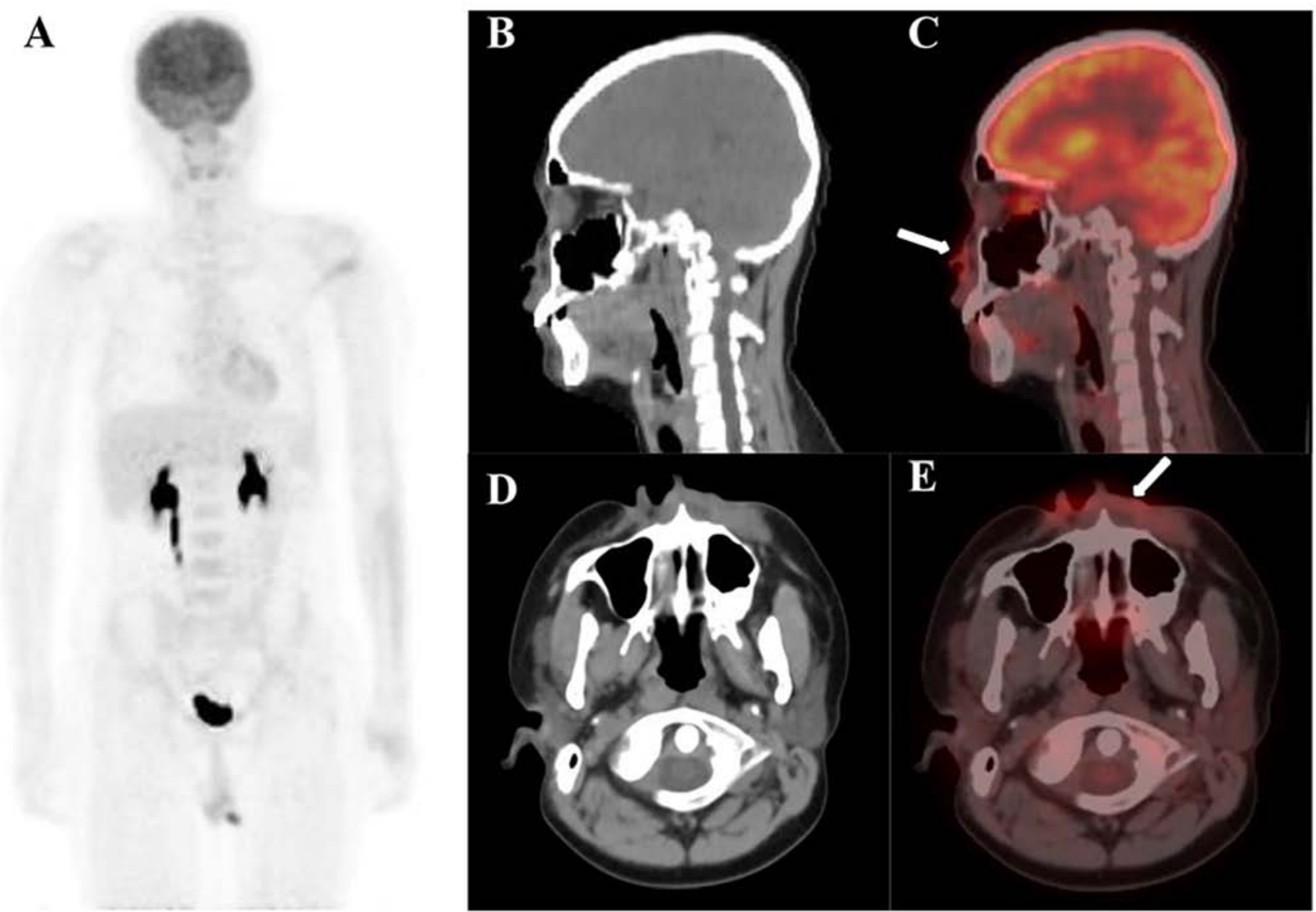

Figure 6. ${ }^{18} \mathrm{~F}-\mathrm{FDG}$ PET/CT imaging in a 15-year-old boy with AML after allo-HSCT. (A) Maximum intensity projection PET image showed no notable abnormal ${ }^{18} \mathrm{~F}$-FDG uptake in the whole body of a 15 -year-old boy with acute myeloblastic leukemia following allogeneic hematopoietic stem cell transplantation. (B and C) Sagittal and (D and E) axial images revealed increased ${ }^{18} \mathrm{~F}-\mathrm{FDG}$ uptake in the soft tissue of left cheek (white arrow). The patient died at 36 months after PET/CT scan. PET, positron emission tomography; ${ }^{18} \mathrm{~F}-\mathrm{FDG},{ }^{18} \mathrm{~F}-2$ '-deoxy-2'-fluorodeoxyglucose.

of ${ }^{18} \mathrm{~F}$-FDG BM uptake in patients with a number of types of solid tumor and found that patients with a low level of ${ }^{18} \mathrm{~F}-\mathrm{FDG}$ BM uptake have a longer OS compared with those with high levels of ${ }^{18} \mathrm{~F}-\mathrm{FDG}$ BM uptake. Abe et al (28) demonstrated that patients with peripheral $\mathrm{T}$ cell lymphoma with BM involvement detected by PET/CT exhibited a significantly shorter OS compared with those without BM involvement, even among patients with negative BM histology. However, certain studies have drawn the opposite conclusion that high levels of ${ }^{18} \mathrm{~F}-\mathrm{FDG}$ $\mathrm{BM}$ uptake have no impact on survival, which is consistent with the results of the present study $(29,30)$. However, none of the aforementioned studies investigated patients with AL. Elevated ${ }^{18} \mathrm{~F}$-FDG BM uptake in patients with AL may be different from that in other patients, because BM is the source of leukemic cells and the primary site of leukemia. Moreover, elevated ${ }^{18} \mathrm{~F}$-FDG BM uptake may be associated with reactive myelopoiesis and inflammation, which occurs more frequently in patients with AL (31).

The spleen is a primary location of extramedullary AL (10). In numerous studies involving patients with a different types of cancer, splenic ${ }^{18} \mathrm{~F}$-FDG uptake has been demonstrated to be an independent prognostic factor for predicting recurrence of cancer or OS $(32,33)$. However, the present study demonstrated that elevated splenic ${ }^{18} \mathrm{~F}$-FDG uptake had no impact on prognosis of patients with AL. It is well known that the spleen functions as a coordinator of immune response, a filter of the circulating blood, and a reservoir for circulating cells and platelets (34). Additionally, the spleen has several responsibilities, including hemoglobin degradation, hematopoiesis and iron recovery and plasma volume regulation (35). For patients with solid tumors, splenic metabolism primarily reflects the systemic inflammatory response to cancer (36). However, splenic metabolism on ${ }^{18} \mathrm{~F}$-FDG PET/CT may represent the complex processes of hematopoiesis, which reflects both systemic inflammation and hematological imbalance (32).

There is debate concerning the prognostic significance of EMD in AL. A number of studies involving patients with AL have reported that EMD is an independent prognostic factor for OS $(37,38)$, whereas other studies have demonstrated that EMD has no impact on prognosis $(39,40)$. EMD in these studies was diagnosed by clinical examination. Since not all extramedullary sites are easily detectable, EMD may have been under-diagnosed. ${ }^{18} \mathrm{~F}$-FDG PET/CT is a sensitive imaging modality for diagnosing EMD in $\operatorname{AL}(7,19)$. Kumar et al $(41)$ compared ${ }^{18} \mathrm{~F}$-FDG PET/CT and CT in terms of response and prognosis of patients with AML with EMD and found that PET/CT can identify more lesions and cases of metabolically progressive disease compared with CT alone, thus affecting management. In accordance with these results, the present study demonstrated that $\mathrm{E}_{\mathrm{N}} \mathrm{E}_{\mathrm{M}} \mathrm{E}_{\mathrm{S}}$ involvement detected by ${ }^{18} \mathrm{~F}-\mathrm{FDG}$ PET/CT could serve as an adverse prognostic factor of patients with AL before and/or after allo-HSCT. 
There are a number of limitations in the present study. First, it was a retrospective study with a relatively small number of patients. Further prospective studies with larger sample size are necessary to confirm the findings of the present study. Second, the time interval between allo-HSCT and PET/CT scans was heterogeneous, ranging from 0-12 months. PET/CT scans before and after allo-HSCT were only performed in seven patients. Since ${ }^{18} \mathrm{~F}-\mathrm{FDG}$ PET/CT is not regularly used in the assessment of leukemia, there are no conclusive data on the optimum interval between allo-HSCT and PET/CT scans. Moreover, the retrospective nature of the present study did not permit regulation of the time interval between allo-HSCT and PET/CT evaluation. Further multiple time point studies are required to identify the optimum time point for PET/CT scans. Finally, not all positive $\mathrm{E}_{\mathrm{N}} \mathrm{E}_{\mathrm{M}} \mathrm{E}_{\mathrm{S}}$ lesions, particularly EMD in lymph nodes, were confirmed by histopathology.

The present data indicated that ${ }^{18} \mathrm{~F}-\mathrm{FDG}$ PET/CT imaging serves a key prognostic role in the evaluation of patients with $\mathrm{AL}$ before and/or after allo-HSCT. $\mathrm{E}_{\mathrm{N}} \mathrm{E}_{\mathrm{M}} \mathrm{E}_{\mathrm{S}}$ involvement detected by ${ }^{18} \mathrm{~F}-\mathrm{FDG}$ PET/CT may identify patients with $\mathrm{AL}$ with an unfavorable outcome. Prospective clinical studies with larger cohorts are required to conclusively define the prognostic role of ${ }^{18} \mathrm{~F}-\mathrm{FDG} \mathrm{PET} / \mathrm{CT}$ in patients with AL treated with allo-HSCT.

\section{Acknowledgements}

Not applicable.

\section{Funding}

The present study was supported by National Natural Science Foundation of China (grant no. 81601522), Medical Youth Talent Project of Jiangsu Province (grant no. QNRC2016749) and Suzhou People's Livelihood Science and Technology Project (grant no. SYS2019038).

\section{Availability of data and materials}

The datasets used and/or analyzed during the present study are available from the corresponding author upon reasonable request.

\section{Authors' contributions}

ZXZ, YYZ and SMD conceived the study and wrote and revised the manuscript. JW, TTZ and JHL reviewed, collected and analyzed the data. BZ, QRL and SMD designed the study and acquired the data. All authors contributed to the drafting of the manuscript. All authors read and approved the final manuscript.

\section{Ethics approval and consent to participate}

The present retrospective study (trial registration no. ChiCTR1900024823) was approved by the Ethics Committee of the First Affiliated Hospital of Soochow University (Suzhou, China; approval no. 2019055), with a waiver of informed consent.

\section{Patient consent for publication}

Not applicable.

\section{Competing interests}

The authors declare that they have no competing interests.

\section{References}

1. Gacha-Garay MJ, Niño-Joya AF, Bolaños NI, Abenoza L, Quintero G, Ibarra H, Gonzalez JM, Akle V and Garavito-Aguilar ZV: Pilot study of an integrative new tool for studying clinical outcome discrimination in acute leukemia. Front Oncol 9: 245, 2019

2. Takahashi K, Wang F, Morita K, Yan Y, Hu P, Zhao P, Zhar AA, Wu CJ, Gumbs C, Little L, et al: Integrative genomic analysis of adult mixed phenotype acute leukemia delineates lineage associated molecular subtypes. Nat Commun 9: 2670, 2018.

3. Lennmyr E, Karlsson K, Ahlberg L, Garelius H, Hulegårdh E, Izarra AS, Joelsson J, Kozlowski P, Moicean A, Tomaszewska-Toporska B, et al: Survival in adult acute lymphoblastic leukaemia (ALL): A report from the Swedish ALL registry. Eur J Haematol 103: 88-98, 2019.

4. Yi M, Li A, Zhou L, Chu Q, Song Y and Wu K: The global burden and attributable risk factor analysis of acute myeloid leukemia in 195 countries and territories from 1990 to 2017: Estimates based on the global burden of disease study 2017. J Hematol Oncol 13: 72, 2020.

5. Malard F and Mohty M: Acute lymphoblastic leukaemia. Lancet 395: 1146-1162, 2020.

6. Hangai M, Urayama KY, Tanaka J, Kato K, Nishiwaki S, Koh K, Noguchi M, Kato K, Yoshida N, Sato M, et al: Allogeneic stem cell transplantation for acute lymphoblastic leukemia in adolescents and young adults. Biol Blood Marrow Transplant 25: 1597-1602, 2019

7. Tan J, Wang Y, Yu SJ, Ma YY, Lei HY and Liu QF: Prognostic factors on graft-versus-host disease-free and relapse-free survival after allogeneic hematopoietic stem cell transplantation for adults with acute leukemia. Leuk Res 59: 1-7, 2017.

8. Halaburda K, Labopin M, Mailhol A, Socié G, Craddock C, Aljurf M, Beelen D, Cornelissen JJ, Bourhis JH, Labussière-Wallet $\mathrm{H}$, et al: Allogeneic stem cell transplantation in second complete remission for core binding factor acute myeloid leukemia: A study from the acute leukemia working party of the European society for blood and marrow transplantation. Haematologica 105: 1723-1730, 2020.

9. Panebianco M, Bagni O, Cenfra N, Mecarocci S, Ortu La Barbera E, Filippi L, Codacci-Pisanelli G, Biondi T, Laghi A and Cimino G: Comparison of 18F FDG PET-CT AND CECT in pretreatment staging of adults with Hodgkin's lymphoma. Leuk Res 76: 48-52, 2019.

10. Zhou WL, Wu HB, Wang LJ, Tian Y, Dong Y and Wang QS: Usefulness and pitfalls of F-18-FDG PET/CT for diagnosing extramedullary acute leukemia. Eur J Radiol 85: 205-210, 2016.

11. Stolzenburg A, Lückerath K, Samnick S, Speer M, Kneer K, Schmid JS, Grigoleit GU, Hofmann S, Beer AJ, Bunjes D, et al: Prognostic value of $\left[{ }^{18} \mathrm{~F}\right] \mathrm{FDG}-\mathrm{PET} / \mathrm{CT}$ in multiple myeloma patients before and after allogeneic hematopoietic cell transplantation. Eur J Nucl Med Mol Imaging 45: 1694-1704, 2018.

12. Qiao W, Zhao J, Wang C, Wang T and Xing Y: Predictive value of (18)F-FDG hybrid PET/CT for the clinical outcome in patients with non-Hodgkin's lymphoma prior to and after autologous stem cell transplantation. Hematology 15: 21-27, 2010.

13. Magnusson E, Cao Q, Linden MA, Frolich J, Anand V, Burns LJ and Bachanova V: Hematopoietic cell transplantation for mantle cell lymphoma: Predictive value of pretransplant positron emission tomography/computed tomography and bone marrow evaluations for outcomes. Clin Lymphoma Myeloma Leuk 14: 114-121, 2014.

14. Döhner H, Estey E, Grimwade D, Amadori S, Appelbaum FR, Büchner T, Dombret H, Ebert BL, Fenaux P, Larson RA, et al: Diagnosis and management of AML in adults: 2017 ELN recommendations from an international expert panel. Blood 129: 424-447, 2017.

15. Brown PA, Wieduwilt M, Logan A, DeAngelo DJ, Wang ES, Fathi A, Cassaday RD, Litzow M, Advani A, Aoun P, et al: Guidelines insights: Acute lymphoblastic leukemia, version 1.2019. J Natl Compr Canc Netw 17: 414-423, 2019.

16. Alam MS, Fu L, Ren YY, Wu HB, Wang QS, Han YJ, Zhou WL, Li HS and Wang Z: 18F-FDG super bone marrow uptake: A highly potent indicator for the malignant infiltration. Medicine (Baltimore) 95: e5579, 2016. 
17. St-Pierre F, Broski SM, LaPlant BR, Ristow K, Maurer MJ, Macon WR, Habermann TM, Ansell SM, Thompson CA, Micallef INM, et al: Detection of extranodal and spleen involvement by FDG-PET imaging predicts adverse survival in untreated follicular lymphoma. Am J Hematol 94: 786-793, 2019.

18. Papajík T, Mysliveček M, Urbanová R, Buriánková E, Kapitáňová Z, Procházka V, Turcsányi P, Formánek R, Henzlová L, Flodr P, et al: 2-[18F]fluoro-2-deoxy-D-glucose positron emission tomography/computed tomography examination in patients with chronic lymphocytic leukemia may reveal richter transformation. Leuk Lymphoma 55: 314-319, 2014.

19. Albano D, Mazzoletti A, Spallino M, Muzi C, Zilioli VR, Pagani C, Tucci A, Rossetti C, Giubbini R and Bertagna F: Prognostic role of baseline 18F-FDG PET/CT metabolic parameters in elderly HL: A two-center experience in 123 patients. Ann Hematol 99: 1321-1330, 2020.

20. Valls L, Badve C, Avril S, Herrmann K, Faulhaber P, O'Donnell J and Avril N: FDG-PET imaging in hematological malignancies. Blood Rev 30: 317-331, 2016.

21. Zhou M, Chen Y, Liu J and Huang G: A predicting model of bone marrow malignant infiltration in ${ }^{18} \mathrm{~F}$-FDG PET/CT images with increased diffuse bone marrow FDG uptake. J Cancer 9: 1737-1744, 2018.

22. Michallet AS, Sesques P, Rabe KG, Itti E, Tordot J, TychyjPinel C, Baseggio L, Subtil F, Salles G, Dupuis JM and Conte MJ: An 18F-FDG-PET maximum standardized uptake value $>10$ represents a novel valid marker for discerning Richter's Syndrome. Leuk Lymphoma 57: 1474-1477, 2016.

23. Stölzel F, Röllig C, Radke J, Mohr B, Platzbecker U, Bornhäuser M, Paulus T, Ehninger G, Zöphel K and Schaich M: ${ }^{18} \mathrm{~F}$-FDG-PET/CT for detection of extramedullary acute myeloid leukemia. Haematologica 96: 1552-1556, 2011.

24. Arslan F, Yilmaz M, Cakir T and Mert A: Significant contribution of Fluorodeoxyglucose positron emission tomography/computed tomography (FDG PET/CT) in a case of acute lymphoblastic leukemia presenting with fever of unknown origin. Intern Med 53: 789-791, 2014.

25. Su K, Nakamoto Y, Nakatani K, Kurihara K, Hayakawa N and Togashi K: Diffuse homogeneous bone marrow uptake of FDG in patients with acute lymphoblastic leukemia. ClinNucl Med 38: e33-e34, 2013

26. Derlin T, Alchalby H, Bannas P, Veldhoen S, Apostolova I, Triviai I, Bengel FM and Kröger N: Assessment of bone marrow inflammation in patients with myelofibrosis: An 18F-fluorodeoxyglucose PET/CT study. Eur J Nucl Med Mol Imaging 42: 696-705, 2015.

27. Jeong SY, Kim SJ, Pak K, Lee SW, Ahn BC and Lee J: Prognostic value of $18 \mathrm{~F}$-fluorodeoxyglucose bone marrow uptake in patients with solid tumors: A meta-analysis. Medicine (Baltimore) 97: e12859, 2018

28. Abe Y, Kitadate A, Usui Y, Narita K, Kobayashi H, Miura D, Takeuchi M, O'uchi E, O'uchi T and Matsue K: Diagnostic and prognostic value of using 18F-FDG PET/CT for the evaluation of bone marrow involvement in peripheral T-cell lymphoma. Clin Nucl Med 44: e336-e341, 2019.

29. Khan AB, Barrington SF, Mikhaeel NG, Hunt AA, Cameron L, Morris T and Carr R: PET-CT staging of DLBCL accurately identifies and provides new insight into the clinical significance of bone marrow involvement. Blood 122: 61-67, 2013.
30. Hong J, Lee Y, Park Y, Kim SG, Hwang KH, Park SH, Jeong J, Kim KH, Ahn JY, Park S, et al: Role of FDG-PET/CT in detecting lymphomatous bone marrow involvement in patients with newly diagnosed diffuse large B-cell lymphoma. Ann Hematol 91: 687-695, 2012.

31. Chen Y, Zhou M, Liu J and Huang G: Prognostic value of bone marrow FDG uptake pattern of PET/CT in newly diagnosed diffuse Large B-cell lymphoma. J Cancer 9: 1231-1238, 2018.

32. Yoon HJ, Kim BS, Moon CM, Yoo J, Lee KE and Kim Y: Prognostic value of diffuse splenic FDG uptake on PET/CT in patients with gastric cancer. PLoS One 13: e0196110, 2018.

33. Kim SY, Moon CM, Yoon HJ, Kim BS, Lim JY, Kim TO, Choe AR, Tae CH, Kim SE, Jung HK, et al: Diffuse splenic FDG uptake is predictive of clinical outcomes in patients with rectal cancer. Sci Rep 9: 1313, 2019.

34. Mebius RE and Kraal G: Structure and function of the spleen. Nat Rev Immunol 5: 606-616, 2005.

35. Sharma Poudel B and Karki L: Abnormal hepatic function and splenomegaly on the newly diagnosed acute leukemia patients. JNMA J Nepal Med Assoc 46: 165-169, 2007.

36. Nam HY, Kim SJ, Kim IJ, Kim BS, Pak K and Kim K: The clinical implication and prediction of diffuse splenic FDG uptake during cancer surveillance. Clin Nucl Med 35: 759-763, 2010.

37. Sakellari I, Gavriilaki E, Batsis I, Mallouri D, Gavriilaki M, Apostolou C, Iskas M, Voutiadou G, Bouziana S, Bousiou Z, et al: Isolated extramedullary relapse as a poor predictor of survival after allogeneic hematopoietic cell transplantation for acute leukemia. Biol Blood Marrow Transplant 25: 1756-1760, 2019.

38. Støve HK, Sandahl JD, Abrahamsson J, Asdahl PH, Forestier E, Ha SY, Jahnukainen K, Jónsson ÓG, Lausen B, Palle J, et al: Extramedullary leukemia in children with acute myeloid leukemia: A population-based cohort study from the nordic society of pediatric hematology and oncology (NOPHO). Pediatr Blood Cancer 64, 2017.

39. Kobayashi R, Tawa A, Hanada R, Horibe K, Tsuchida M and Tsukimoto I; Japanese childhood AML cooperative study group: Extramedullary infiltration at diagnosis and prognosis in children with acute myelogenous leukemia. Pediatr Blood Cancer 48: 393-398, 2007.

40. BisschopMM,Révész T, Bierings M,van Weerden JF, van Wering ER, Hählen K and van der Does-van den Berg A: Extramedullary infiltrates at diagnosis have no prognostic significance in children with acute myeloid leukaemia. Leukemia 15: 46-49, 2001.

41. Kumar R, Harish N, Sharma A, Gupta RK, Sharma A, Bakhshi S, Patel C, Thankarajan AS and Bal C: Comparison of FDG $\mathrm{PET} / \mathrm{CT}$ and $\mathrm{CT}$ in response assessment and prognosis of patients with extra medullary disease in acute myeloid leukemia. J Nucl Med 60 (Suppl 1): S1251, 2019.

(i) 9 This work is licensed under a Creative Commons Attribution-NonCommercial-NoDerivatives 4.0 International (CC BY-NC-ND 4.0) License. 